\title{
Shape optimal design of phases in composites: harmonic problem
}

\author{
P. P. Prochazka \\ Civil Engineering, Czech Technical University in Prague, \\ Czech Republic
}

\begin{abstract}
In the paper identification of phases micro-geometry is studied providing that the overall properties are given as well as the intrinsic characteristics of the phases. Harmonic problem is solved, so that the linear steady-state conductivity problem is a typical application to be discussed. For example, overall change of temperature and the temperature characteristics are given as well as the set of all conductivities are prescribed. The objective of the solution is the shape of phases fulfilling the condition that the homogenized and given values of the conductivity tensors are as close as possible in some sense. This task appears as non-solvable uniquely and even can exceed the realistic results. This is why additional constraints have to be added. One of such reasonable conditions is the restriction on the volume (in 2D the area) of phases involved in the composite structure. Still, this cannot be sufficient to meet a realistic situation, since the side condition is mostly formulated in integral form, i.e. positive and negative signs can lead to non-realistic geometry. For this reason, restrictions on the shape of phases should be introduced, such as the diameters or tangential inclination of certain directions of the phase boundaries.

Practical effects are of great importance to engineers dealing with composite materials (aerospace, civil, mechanical engineers, and similar). This optimization is enabled by having one free property: the shape of the phases. The mathematical formulation and subsequent numerical treatment utilizes this opportunity and provides a reasonable, fully usable in practice, layout.

Keywords: composite structure, shape optimization, micro- and macro-levels.
\end{abstract}




\section{Introduction}

The concept of composite media stems not only from the mechanical background but also provides a theoretical means for relaxation of variational principles. This is a typical result of the localization and homogenization theories of composites, which, as best way of formulation of physical problems, starts with equality of energies at both the macro- and the micro-level. In this case, the results in both these levels are dependent on the geometry of the phases. As mentioned in many publications $[1,2]$, there are many approaches on how to solve this problem. Hereinafter similar procedure as that used in [3] is applied for formulating and calculating the proper shape of phases. Time dependent and steady state problems of heat transfer in composites are solved in [4], for example.

It is worth noting a brilliant idea of Levin who put forward fundaments of homogenization in coupled problem of elasticity and heat transfer [5]. Classical approach in localization and homogenization of elastic composites belongs to Suquet, [6] and that of steady state heat transfer can be found also in [7], which is applied to periodic composites. The meaning "periodic structure" is explained for periodic representative element. More or less this procedure is applied in this paper to unit cell concept.

In this text the steady-state problems, such as heat and mass density transfer, filtration of the Newtonian liquid, etc., are considered. First, homogenization technique will be suggested and a variational formulation will characterize the problem, so that finite elements can easily be derived. Second, cost functionals will be created and provide designers with a range of possible conditions for their request.

\section{Basic considerations and equations}

In this paper $2 \mathrm{D}$ problem of steady state heat transfer in composite structure will be treated. Another one assumption is introduced: two-phase composite is taken into account with one phase fiber and the other matrix, both in a periodic cell, which is cut out of a representative volume element $V$ describing the neighborhood of a typical point of macrostructure.

\subsection{Periodic boundary conditions}

Since the periodic boundary conditions are of great importance in each composite structure, they will briefly be discussed in this section. Representative volume element $(R V E)$ is cut from the whole body under consideration and is assumed as a representative of geometrical and mechanical properties at a given location. $R V E$ typically used in this work is depicted in Fig. 1.

Let the body representing the composite is denoted as $\Omega \subset V \in R^{2}$ and its boundary $\partial \Omega$ is supposed to be Lipschitz continuous. The latter condition will be fulfilled implicitly, as the shape of the unit cell $\Omega$ is a square $(0,1) \times(0,1)$. Two isotropic phases $\Omega_{\mathrm{f}} \subset \Omega$ and $\Omega_{\mathrm{m}} \subset \Omega$ represent fiber and matrix. The 
boundary of the fiber (i.e. the interfacial boundary $\Gamma_{\mathrm{C}}$ ) is star shaped. Although the fibers are generally placed in the body in a disordered way, certain reasonable arrangement is necessary to contemplate. Fig. 1 offers a possible layout used in what follows. It leads to rectangular or square unit cell, Fig. 2. Note that more general shapes are mentioned in [8], where a special treatment on how to simplify complicated unit cells is also discussed based on body transformation. The transformations create a group of base bodies.

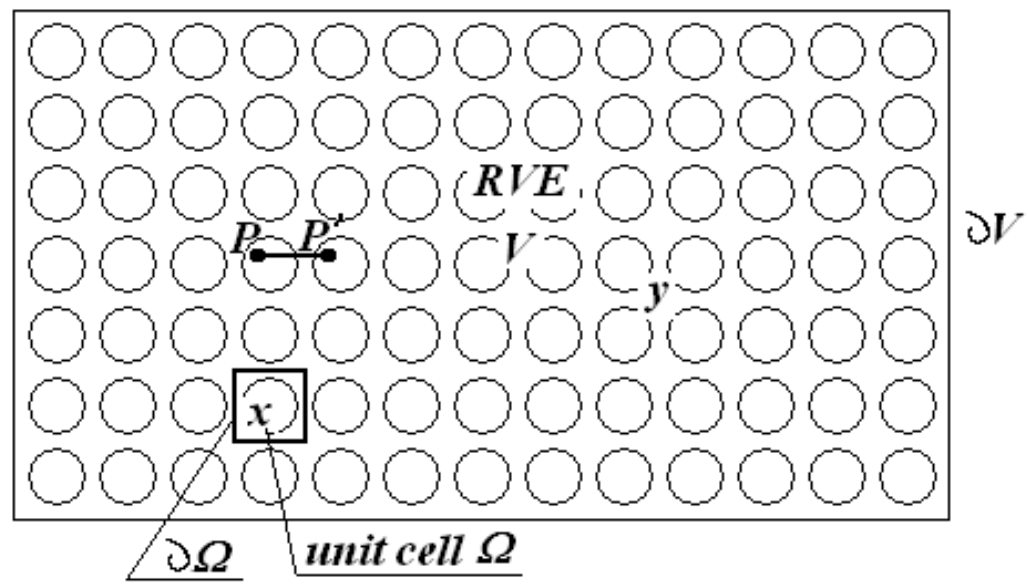

Figure 1: $\quad$ Layout inside $R V E$ and brief denotation.

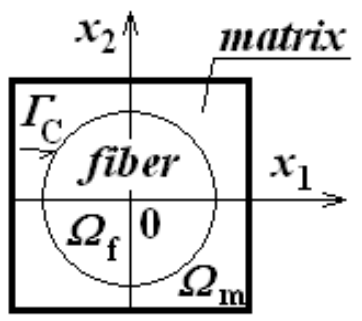

Figure 2: Unit cell.

In $R V E$ coordinate system $0 y_{1} y_{2}$ is introduced while the unit cells are equipped by local coordinate system $0 x_{1} x_{2}$, as $2 \mathrm{D}$ problem is discussed.

Now the periodic conditions will be précised. The unit cells in the $R V E$ are homothetic, i.e. there is a constant, say $\ell$, and points like $P$ and $P^{\prime}$ in Fig. 1 are identified by the law: $P^{\prime}(y)=P(y+\ell)=\ell \overrightarrow{P P^{\prime}}$ and the function to be considered as periodic has exactly the same value at $P$ and $P^{\prime}$. In our case a square unit cell is taken into account. Hence the homothetic property is applicable in both $y_{1}$ 
and $y_{2}$ directions. Denote $L$ the macroscopic length, it means that this is a characteristic length of $R V E$.

The periodicity can also be defined in the following way: take a small number $\varepsilon=\ell / L$, consequently, $\Omega=\varepsilon V$.

\subsection{Formulation}

The conservation law is considered in the standard divergence form applied to temperature $u^{\varepsilon}(x)$ as,

$$
-\frac{\partial}{\partial x_{i}}\left(c^{\varepsilon} \frac{\partial}{\partial x_{i}} u^{\varepsilon}\right)=0
$$

where $c^{\varepsilon} \equiv c^{\varepsilon}(x)$ is dependent on the position in $\Omega$, consisting of two subdomains, fiber and matrix, which are equipped by different conductivity values $a$ (fiber) and $b$ (matrix), where $a$ and $b$ are constants. This means that the coefficient of conductivity $c^{\varepsilon}(\boldsymbol{x})$ is defined as:

$$
c^{\varepsilon}(\boldsymbol{x})=b_{\mathrm{f}} \quad \text { for } \quad \boldsymbol{x} \in \Omega_{\mathrm{f}} \quad \text { and } \quad c^{\varepsilon}(\boldsymbol{x})=b_{\mathrm{m}} \quad \text { otherwise }
$$

or

$$
c^{\varepsilon}(x)=a \chi(x)+b(1-\chi(x))
$$

where $\chi$ is the characteristic function of the fiber.

The partial equations are as

$$
\nabla \boldsymbol{q}^{\varepsilon}=0, \quad \boldsymbol{q}^{\varepsilon}=-c^{\varepsilon} \nabla u^{\varepsilon}
$$

where $u^{\varepsilon}$ is still the temperature (it can involve also other quantities, which are mentioned in the introduction, $\nabla$ is the nabla operator, and $\boldsymbol{q}^{\varepsilon}$ is the flux vector. Eqns. (3) describe the steady-state conditions of processes such as the heat or mass transfer, the electric conductivity and permittivity, filtration of a Newtonian liquid and such. It is a transfer equation (Fourier, Ficks, Ohm, Darcy, etc.). In the case of ideal (or perfect) interfacial contact $\Gamma_{C}$ between the fiber and matrix it can be introduced the normal component of the flux $q_{\mathrm{n}}(\boldsymbol{x})=q(\boldsymbol{x}) n(\boldsymbol{x})$.

The flux and potential $u(x)$ have to fulfill the interface conditions, namely the continuity across the interface of both phases. The unit normal $n(\boldsymbol{x})$ is defined as $n(\boldsymbol{x}) \perp \partial \Omega_{\mathrm{f}}$. The interfacial conditions are appended with boundary conditions on $\partial \Omega$, where either

$$
T(\boldsymbol{x})=\boldsymbol{P} \cdot \boldsymbol{x} \quad \text { or } \quad q_{\mathrm{n}}(\boldsymbol{x})=Q(\boldsymbol{x}) \cdot n(\boldsymbol{x}) \quad \text { on } \partial \Omega
$$

holds for $\boldsymbol{x} \in \partial \Omega$. Symmetric matrices $\boldsymbol{P}$ and $\boldsymbol{Q}$ are constant symmetric matrices given for a specific problem. 
For statistically homogeneous material with the periodic boundary conditions or conditions given by Eqns. (4) an analog of the well known Hill condition holds valid as:

$$
\begin{aligned}
& <\boldsymbol{q} \nabla u>=\frac{1}{\text { meas } \Omega} \int_{\Omega} \boldsymbol{q} \nabla u \mathrm{~d} \Omega(\boldsymbol{x})= \\
& =<\boldsymbol{q}><\nabla u>=\frac{1}{\operatorname{meas} \Omega} \int_{\Omega} \boldsymbol{q} \mathrm{d} \Omega(\boldsymbol{x}) \times \frac{1}{\operatorname{meas} \Omega} \int_{\Omega} \nabla u \mathrm{~d} \Omega(\boldsymbol{x})
\end{aligned}
$$

where meas $\Omega$ is the volume in $3 \mathrm{D}$ or area in $2 \mathrm{D}$, mostly considered equal to one (for this reason one speaks about unit cell $\Omega$ ). As a consequence of Eqn. (6) for overall conductivity $c^{*}$ it immediately follows:

$$
<\boldsymbol{q}>=-c^{*}<\nabla u>, \quad\left\langle\boldsymbol{q} \nabla u>=-c^{*}<q>\langle\nabla u>\right.
$$

It is sometimes also of interest to us to introduce the resistivity $C=c^{-1}$. Then similarly to (6) it is:

$$
<\nabla u>=-C^{*}<\boldsymbol{q}>, \quad\left\langle\boldsymbol{q} \nabla u>=-C^{*}<\boldsymbol{q}><\nabla u>\right.
$$

Exactly, as in case the elasticity problem is solved concentration factors $\boldsymbol{A}(\boldsymbol{x})$ and $\boldsymbol{B}(\boldsymbol{x})$ are introduced to relate the overall conductivity and resistivity as:

$$
c^{*}=<c(\boldsymbol{x}) \boldsymbol{A}(\boldsymbol{x})>, \quad C^{*}=<c(\boldsymbol{x}) \boldsymbol{B}(\boldsymbol{x})>
$$

The above relations are prevailingly useful for optimization expressed as classical variational principles.

\section{Homogenization}

In order to simplify the problem square unit cell and by phases uniform coefficient of conductivity are supposed in this paper. In order to get the relations between local and overall coefficient of conductivity an asymptotic expansion of $u^{\varepsilon}$ and $q^{\varepsilon}$ will be considered for small enough $\varepsilon$ :

$$
\begin{aligned}
& u^{\varepsilon}(x)=u_{0}(x, y)+\varepsilon u_{1}(x, y)+\ldots \\
& \boldsymbol{q}^{\varepsilon}(x)=\boldsymbol{q}_{0}(x, y)+\varepsilon \boldsymbol{q}_{1}(x, y)+\ldots \quad y=x / \varepsilon
\end{aligned}
$$

where $u_{i}$ and $\boldsymbol{q}_{i}$ are $\Omega$-periodic in $y$. In what follows coordinates $x$ and $y$ are first taken as independent and afterwards $y$ is substituted by $x / \varepsilon$. Since only 
differentiation of the first order is applicable in Eq. (1), operator $\frac{\partial}{\partial x_{i}}$ is now read as $\frac{\partial}{\partial x_{i}}+\frac{1}{\varepsilon} \frac{\partial}{\partial y_{i}}$. Substituting Eq. (9) to Eq. (1) yields at $\mathrm{O}\left(\varepsilon^{-2}\right)$ :

$$
-\frac{\partial}{\partial y_{i}}\left(c^{0} \frac{\partial}{\partial y_{i}} u_{0}\right)=0
$$

It can easily be shown that the only periodic solution of Eq. (10) is constant in $y$, i.e.

$$
u_{0}(x, y)=u_{0}(x)
$$

From Eq. (1) at $0\left(\varepsilon^{-1}\right)$ and with respect to Eq. (10) yields:

$$
-\frac{\partial}{\partial y_{i}}\left[c^{0}\left(\frac{\partial u_{1}}{\partial y_{i}}+\frac{\partial u_{0}}{\partial x_{i}}\right)\right]=0
$$

which is the starting equation for $u_{1}$ with $u_{0}$ given. Eq. (12) is an elliptic equation being valid in $\Omega$. If comparing with the elasticity problem $\frac{\partial u_{0}}{\partial x_{i}}$ is a unit impulse and $u_{1}$ is a fluctuating term. Since also the Laplace equation (1) is linear, similar approach to that used in linear elasticity can also be applied hereinafter.

In Eq. (12) $u_{0}$ is supposed to be known and this equation is elliptic one for unknown $u_{1}$ being valid in $\Omega$ and is $\Omega$-periodic.

In order to get the formulation in finite elements introduce space $V \equiv\left\{u \in H_{p e r}^{1}(\Omega)\right.$, Lebesque messure over $\Omega$ of $u$ disappears $\}$ Variational formulation of Eq. (12) can be reached by multiplying both sides of Eq. (12) by test function $\varphi \in V$ and integrate over $\Omega$ to get: find $u_{1} \in V$ such that it holds for each $\varphi \in V$ :

$$
\int_{\Omega} a(y) \frac{\partial u_{1}}{\partial y_{i}} \frac{\partial \varphi}{\partial y_{i}} \mathrm{~d} y=-\frac{\partial u_{0}}{\partial y_{i}} \int_{\Omega} a(y) \frac{\partial \varphi}{\partial y_{i}} \mathrm{~d} y
$$

Let us solve an auxiliary problem: find function $u_{1} \in V$ so that

$$
\int_{\Omega} a(y) \frac{\partial w}{\partial y_{i}} \frac{\partial \varphi}{\partial y_{i}} \mathrm{~d} y=-\int_{\Omega} a(y) \frac{\partial \varphi}{\partial y_{i}} \mathrm{~d} y
$$


is fulfilled for all $\varphi \in V$. Because of linearity it immediately follows:

$$
u_{1}=\frac{\partial u_{0}}{\partial x} w
$$

Eqs. (9) and (12) yield:

$$
\boldsymbol{q}_{0}(\boldsymbol{x}, \boldsymbol{y})=c^{0}\left(\frac{\partial u_{1}}{\partial y_{i}}+\frac{\partial u_{0}}{\partial x_{i}}\right)
$$

By virtue of Eqs. (15) and (16) and by the constitutive relation it holds:

$$
q_{0}=a_{h} \frac{\partial u_{0}}{\partial x}, \quad \text { where } \quad a_{h}=\int_{\Omega} a(y)\left(1+\frac{\partial w}{\partial y}\right) \mathrm{d} y
$$

where the supposition of symmetry in both geometry and distribution of conductivity coefficients is taken into account.

\section{Optimization}

Recall that the trial problem has been formulated as for given overall conductive coefficient $A$ it is required such a shape of fiber $\Omega^{\mathrm{f}}$ for which the square of subtraction $\left(A-a_{h}\right)^{2}$ should be minimum under additional constraint concerning constant volume of fiber meas $\Omega^{\mathrm{f}}$. Using Lagrangian multiplier $\lambda$ the problem can be established as:

$$
\Pi\left(a, \Omega^{\mathrm{f}}\right)=\frac{1}{2}\left(A-a_{h}\right)^{2}-\lambda\left(\int_{\Omega^{\mathrm{f}}} d \Omega-\text { meas } \Omega^{\mathrm{f}}\right) \rightarrow \text { stationary }
$$

and only the calculated coefficient of conductivity is dependent on the shape of fiber.

This shape will be identified by radii $p_{\mathrm{s}}, s=1,2, \ldots, n$ of nodes located at the interface $\Gamma_{C}$ connecting the origin of the local coordinate system lying at the lower left vertex of the unit cell, Fig. 2.

Without lack of generality, let us consider a symmetric unit cell depicted in Fig. 2. With respect to the symmetry instead of Eq. (14) it is to solve

$$
\int_{\Omega^{\mathrm{f}}} a(y) \frac{\partial w}{\partial y} \frac{\partial \varphi}{\partial y} \mathrm{~d} y=-\int_{\Omega^{\mathrm{f}}} a(y) \frac{\partial \varphi}{\partial y} \mathrm{~d} y
$$

and $a_{h}$ follows from Eq. (17).

It remains to specify the domain $\Omega^{\mathrm{f}}$ by means of its corresponding boundary. This can be done in many ways. Hereinafter suppose the polygonal shape of the fiber under study approximating the continuous shape. One can choose some 
fixed point (pole - in our case this is the origin) and connect it with each vertex of this polygonal boundary, the distance of the $i$-th vertex from the origin of the coordinate system is denoted as $p_{i}$. In this way we obtain $n$ triangles $T_{s}, s=$ $1, \ldots, n$. It obviously holds:

$$
\int_{\Omega^{\mathrm{f}}} d \Omega=\text { meas } \Omega^{\mathrm{f}}=\sum_{s=1}^{n} \text { meas } T_{s} .
$$

In most cases of fiber volume ratio, combination of phase conductivities and given overall conductivity certain restrictive conditions have to be applied to the admissible beams of nodes at the interfacial boundary. This can be done in various ways. In this paper the lowest value of the length of any node at $\Gamma_{C}$ is bounded from below by a given value and the highest length is constrained by the conditions as:

$$
y_{1} \leq p_{1}<1, \quad y_{2} \leq p_{2}<1
$$

and $p_{1}$ and $p_{2}$ are given in advance.

If the above bounds on the beams are attained a special procedure needs to be used, see [3]. It requires an internal iteration, as the improvement of the boundary using collinear mapping to ensure the condition about constant fiber volume fraction.

It is worth noting that other constraint conditions can be applied, see e.g. [3]. They are applicable also here. The procedure suggested in this paper is based on finite element method while in [3] the numerical tool is based on boundary elements. The difference is in the choice of $p_{1}, p_{2}$, which in this paper should be much lesser then 1 and can be closer to 1 in case the boundary element method is applied.

\subsection{Euler's equations}

The stationary requirement leads to differentiation of the functional by the shape (design) parameters $p_{s}$

$$
\frac{\partial \Pi\left(a_{h}, \Omega\right)}{\partial p_{s}}=\left(A-a_{h}(\boldsymbol{p})\right) \frac{\partial a_{h}(\boldsymbol{p})}{\partial p_{\boldsymbol{s}}}+\lambda \frac{\partial}{\partial p_{s}} \int_{\Omega^{\mathrm{f}}} \mathrm{d} \Omega(\boldsymbol{p})=0
$$

which can be rewritten as:

$$
E_{s}+\lambda=0, \quad s=1,2, \ldots, n
$$

where 


$$
E_{s}=-\frac{\left(A-a_{h}\right) \frac{\partial a_{h}(\boldsymbol{p})}{\partial p_{\boldsymbol{s}}}}{\frac{\partial}{\partial p_{s}} \int_{\Omega^{\mathrm{f}}} \mathrm{d} \Omega(\boldsymbol{p})} \quad \text { for each } s=1, \ldots, n
$$

Equation (24) requires $E_{s}$ to have the same value for any $s$. In other words, if this requirement is attained at any point on the "moving" part of the interfacial boundary the optimal shape of the trial body would be reached. For this reason the body of the composite structure should increase its area (in 3D its volume) at the nodal point $p_{s}$ of the boundary, if $E_{s}$ is larger than the true value of $-\lambda$, while it should decrease its value when $E_{s}$ is smaller than the correct $-\lambda$. As, most probably, real value $-\lambda$ is not known a priori, its estimate is done by averaging the current values at the nodal points. So, approximation of $\lambda$ will be expressed as:

$$
\lambda_{\text {approx }}=-\frac{1}{n} \sum_{i=1}^{n} E_{S}
$$

Differentiation by $\lambda$ completes the system of Euler's equations by Eq. (20).

It remains to ensure that the fiber volume friction is constant with the value given a priori. For this aim a collinear mapping is applied after completing the shift of nodes at the interface. It can be done in such a way that assuming the current value of meas $\Omega_{\text {curr }}^{\mathrm{f}}$, which is calculated from the current positions of the nodes mentioned, the prescribed meas $\Omega^{\mathrm{f}}$ is reached by improving the triangles $T_{i}$ by the value of

$$
s=\sqrt{\frac{\text { meas } \Omega_{\text {curr }}^{\mathrm{f}}}{\text { meas } \Omega^{\mathrm{f}}}}
$$

Brief description of algorithm:

1 set up the starting configuration fulfilling the condition given by Eq. (20)

2 calculate $a_{h}$ for the current configuration

3 set a successive unit shifts to nodal points $p_{s}$ at $\Gamma_{C}$ and calculate $a_{h}\left(p_{s}\right)$ and $E_{S}$ by Eq. (24) using substitution of derivatives by differences

4 compute $\lambda_{\text {approx }}$ by Eq. (25) to get new positions of nodes at $\Gamma_{C}$

5 from the new positions get meas $\Omega_{\text {curr }}^{\mathrm{f}}$

6 using collinear mapping improve the positions of nodes by Eq. (26) to ensure the original fiber volume ratio 
7 check up the constraint of the beams $p_{s}$ and if fail occurs apply local iteration

8 Euclidean distance between current and previous energies $E_{S}$ should be less then given admissible error; if not, go to 2 and stop otherwise

\section{Example}

Unit cell is considered with various fiber volume ratios. Since we compare energy densities at nodal points of the interfacial boundary, the relative energy density may be regarded as the comparative quantity influencing the movement of the boundary $\Gamma_{C}$. As said in the previous section, the higher value of this energy, the larger movement of the nodal point of $\Gamma_{C}$ should aim at the optimum. The process of iterations will end if the Euclidean distance between current and previous energies be less then given admissible error.

In the following examples required conductivity $A$ is given, the fiber and matrix phase ratios are also given as:

$$
c_{\mathrm{f}}=\text { meas } \Omega^{\mathrm{f}}, \quad c_{\mathrm{m}}=\text { meas } \Omega^{\mathrm{m}}
$$

and also the conductivities $b_{\mathrm{f}}$ and $b_{\mathrm{m}}$ are prescribed.

In the first numerical test it is: $c_{\mathrm{f}}=40 \%, c_{\mathrm{m}}=60 \%, b_{\mathrm{f}}=1, b_{\mathrm{m}}=5$, target $A=3, a=3$, the relative error is $3.8 \mathrm{e}-04$ after seven iterations using the step of iteration 0.1 . The result is depicted in Fig. 3.

For the next case $c_{\mathrm{f}}=60 \%, c_{\mathrm{m}}=40 \%, b_{\mathrm{f}}=1, b_{\mathrm{m}}=5$, target $A=2.7$, $a=2.7$, the relative error is $3.3 \mathrm{e}-04$ after ten iterations using the step of iteration 0.1 . The result is depicted in Fig. 4.

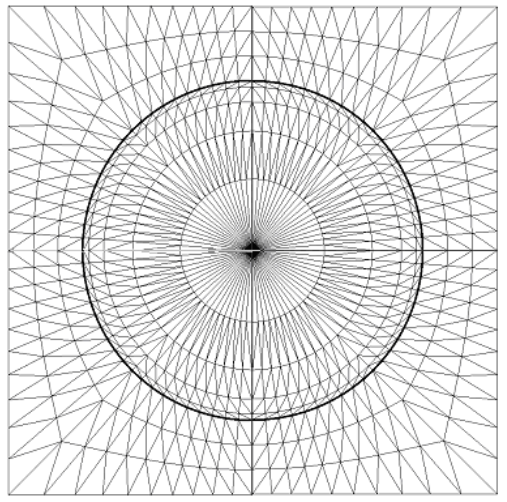

Figure 3: $\quad$ First case.

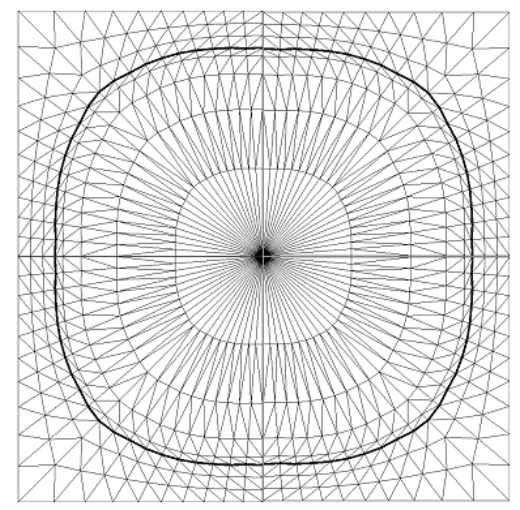

Figure 4: $\quad$ Second case.

The previous cases have not required respecting any restrictions on the length of beams. The last examples have to involve the local iteration, as the beams are 
bounded by 0.05 from the external boundary $\partial \Omega$. The properties are as: $c_{\mathrm{f}}=50 \%, c_{\mathrm{m}}=50 \%, b_{\mathrm{f}}=1, b_{\mathrm{m}}=5$, target $A=3, a=3$, the relative error is $2.57 \mathrm{e}-03$ after twenty iterations using the step of iteration 0.1 . The result is seen in Fig. 5. Fig. 6 shows the results from the same constellation but $c_{\mathrm{f}}=40 \%$ and $c_{\mathrm{m}}=60 \%$. Twenty seven iterations had to be carried out and the error is $2.51 \mathrm{e}-$ 2.

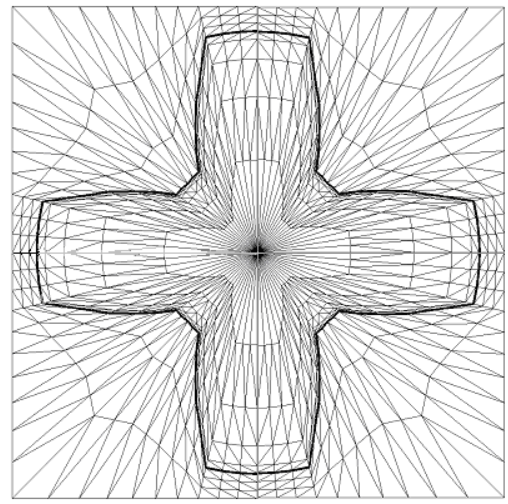

Figure 5: $\quad$ First case.

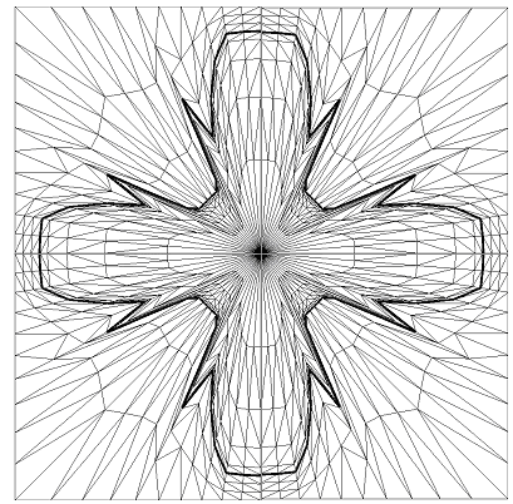

Figure 6: Second case.

The results show certain shapes of fibers which can be used in practice. The last case only shows possible direction on how to construct fiber, and while the previous cases are quite reasonable the last case is more or less scholar.

\section{Acknowledgement}

Financial support of the Grant agency of the Czech Republic, grant number $\mathrm{P} 105 / 00 / 0266$ is gratefully acknowledged.

\section{References}

[1] V.J. Challis, A.P. Roberts, A.H. Wilkins: Design of three dimensional isotropic microstructures for maximized stiffness and conductivity. Int. J. Solids and Structures, 45, (14-15), 2008, 4130-4146.

[2] E.M. Dede: Simulation and optimization of heat flow via anisotropic material thermal conductivity. Comput. Mat. Sci., 50, (2), 2010, 510-515.

[3] P.P. Prochazka, V. Dolezel, T.S. Lok: Optimal shape design for minimum Lagrangian. Eng. Anal. With Bound. Elem. 33, 2009, 447-455.

[4] M. Kamiński: Homogenization of transient heat transfer problems for some composite materials. Int. J. Eng. Sci., 41, (1), 2003, 1-29.

[5] I.M. Levin: Thermal expansit coefficients of heterogeneous materials. Kvestii Akademii Nauk SSSR, Mechanika Tverdogo Tela, 1967, 88-94. 
[6] P.M. Suquet: Homogenization techniques for composite media. Lecture, Notes in Physics 272 (eds. E. Sanches-Palencia and A. Zaoi) Part IV, Sprinter Verlag Berlin, 1985, 194-278.

[7] T. Lévy: Fluids in porous media and suspensions. Lecture Notes in Physics 272 (eds. E. Sanches-Palencia and A. Zaoi) Part II, Sprinter Verlag Berlin, 1985, 64-119.

[8] J. Dvorak: Optimization of composite materials, $\mathrm{PhD}$ thesis, Charles University, June 1996. 